La revue La revue pour l'histoire du CNRS

POUR LHISTORE DU CNRS $\quad 25 \mid 2010$

L'Aventure européenne du CNRS, I

\title{
Le budget du CNRS de la Libération à 1968.
}

Bruno Marnot

\section{(2) OpenEdition}

Journals

Édition électronique

URL : https://journals.openedition.org/histoire-cnrs/9247

DOI : 10.4000 /histoire-cnrs.9247

ISSN : 1955-2408

Éditeur

CNRS Éditions

Édition imprimée

Date de publication : 15 octobre 2010

Pagination : 38-42

ISBN : 978-2-271-07114-9

ISSN : 1298-9800

Référence électronique

Bruno Marnot, « Le budget du CNRS de la Libération à 1968. ", La revue pour l'histoire du CNRS [En ligne], 25 | 2010, mis en ligne le 15 octobre 2012, consulté le 20 mai 2021. URL : http://

journals.openedition.org/histoire-cnrs/9247 ; DOI : https://doi.org/10.4000/histoire-cnrs.9247

Ce document a été généré automatiquement le 20 mai 2021.

Comité pour l'histoire du CNRS 


\title{
Le budget du CNRS de la Libération à 1968.
}

\author{
Bruno Marnot
}

1 Entre la Libération et la fin du XX $\mathrm{XX}^{\mathrm{e}}$ siècle, le CNRS est devenu un organisme immense, complexe, d'envergure internationale. Dans la mesure où il a eu pour ambition de représenter tous ou presque tous les "fronts de la science", son développement a nécessité des moyens financiers croissants. Établissement public, le CNRS vit pour l'essentiel des subventions de l'État, donc du budget de la nation' ${ }^{1}$. L'évolution de ce dernier a, à sa manière, circonscrit des phases bien précises de l'histoire de l'organisme, à la fois en influençant son développement et en étant le reflet de ses orientations scientifiques, mais aussi de ses tendances de longue durée. Quatre périodes ont pu ainsi être identifiées.

\section{Un décollage financier incertain (1944-1958)}

2 À la Libération, le CNRS ne fait évidemment pas partie des priorités gouvernementales dans une conjoncture encore marquée par les pénuries de tous ordres et la nécessité de reconstruire l'économie du pays. Cette situation vaut d'ailleurs pour l'ensemble de la recherche publique française. Parti d'à peu près rien en 1944, le budget du CNRS connaît un taux de croissance extrêmement rapide au cours des années suivantes. Exprimé en francs courants, il se situe à hauteur de 139,7 millions de francs en 1944 pour atteindre 1,735 milliards dès 1948 ! Ce bond en volume prodigieux ne doit pas faire oublier toutefois qu'il intervient dans un contexte de très forte inflation et de profonde instabilité monétaire. La conversion du budget en francs constants apparaît en l'occurrence particulièrement significative ${ }^{2}$. Entre les deux mêmes dates, l'augmentation se situe à hauteur de $131,7 \%$, soit dix fois moins que dans le premier cas de figure. Alors que l'indice des prix à la consommation est multiplié par 5,72 entre 1944 et 1948, le budget du CNRS augmente à un rythme moitié moindre. L'effort n'est donc exceptionnel qu'en apparence. 
Budget du CNRS de 1944 à 1958

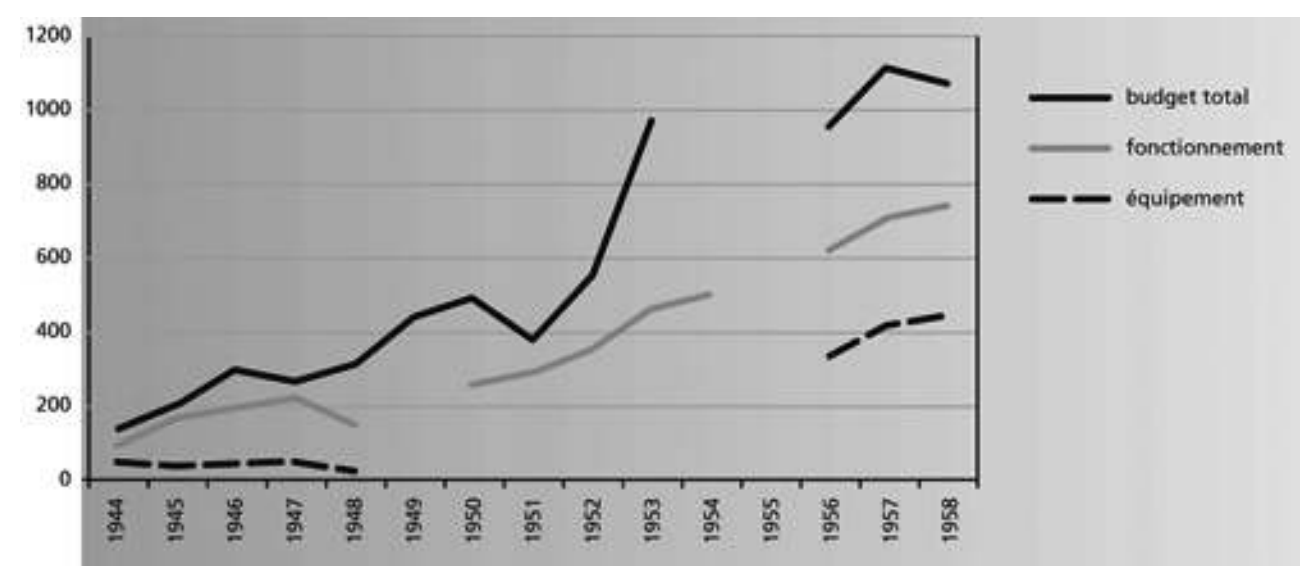

(en millions de francs 1999)

3 En outre, la progression du budget n'est pas régulière, ce qui est préjudiciable aux investissements lourds que doit effectuer l'organisme. Les dirigeants se plaignent constamment de cette situation, comme en 1948 lorsque «tous les crédits demandés pour des travaux de construction ou pour l'équipement nous ont été refusés et [que] nous avons dû renoncer à poursuivre un plan d'édification de nouveaux laboratoires longuement préparé $»^{3}$. L'évolution financière en dents de scie explique en grande partie les limites de l'effort scientifique du Centre.

Cette évolution budgétaire, pour irrégulière qu'elle soit, ne doit pas faire oublier deux caractéristiques essentielles : elle est, d'une part, globalement orientée à la hausse sur l'ensemble de la période, et elle marque, d'autre part, une inflexion au début des années 1950, plus particulièrement à partir du bond de 1952 et plus encore de 1953 qui constituent un palier. Cet élan financier est contemporain du lancement du $\mathrm{II}^{\mathrm{e}} \mathrm{Plan}$ qui intègre les sciences et les techniques. Les dépenses en faveur du personnel, d'une part, l'équipement des laboratoires en matériels et les opérations immobilières, d'autre part, qui correspondent au potentiel de production scientifique du Centre, représentent les trois priorités des années 1945-1958. De ces trois postes, celui relatif aux dépenses de personnel devient rapidement prépondérant dans les finances de l'organisme. Sa proportion par rapport au budget de fonctionnement passe de 37,5\% en 1944 à $46,6 \%$ en 1947, puis au-delà de $75 \%$ à partir de 1950 . Elle redescendra rarement en deçà de ce seuil par la suite. C'est une tendance lourde de l'institution qui s'installe dès ces années.

5 L'importance croissante de la masse salariale entrâne un autre déséquilibre plus profond et durable de la structure budgétaire du Centre, qui concerne la répartition des crédits entre " dépenses ordinaires » et " dépenses extraordinaires » ${ }^{4}$. Du fait même des pesanteurs inhérentes aux premières, le budget de fonctionnement est appelé à croître avec une certaine inertie, ce qui n'est pas forcément le cas des crédits d'équipement, susceptibles de faire davantage les frais des périodes de restriction budgétaire. Trois raisons expliquent l'importance croissante des «dépenses ordinaires». Le réajustement régulier des salaires au coût de la vie, bien sûr, mais aussi «la conséquence de textes accordant des améliorations de situation aux différentes catégories de personnel de l'Etat $\|^{5}$ expliquent cette situation. La raison majeure réside néanmoins dans la croissance exceptionnelle de l'effectif, qui passe de 1080 agents en 1944 à 5972 en 1958, soit une multiplication par 5,5 du nombre d'individus rétribués par le Centre. 
6 En revanche, l'évolution des dépenses consacrées à l'extension des activités scientifiques demeure modeste. Les dépenses de matériel sont logiquement privilégiées, mais les crédits distribués sont partagés entre laboratoires propres et laboratoires extérieurs. Ces derniers bénéficient néanmoins d'un volume plus élevé de crédits et surtout la progression de la manne qui leur échoit est forte et régulière à partir de 1948. Institut scientifique, le CNRS continue aussi d'assumer pleinement sa vocation de "caisse» de recherche. L'étude des subventions globales (personnel et matériel) allouées par le CNRS fait davantage prendre conscience de ce fait. Celles-ci s'élèvent en 1953 à 2,039 milliards de francs, soit la moitié du budget total du Centre.

Évolution de différents crédits accordés au CNRS (millions de francs)

\begin{tabular}{cccccc} 
& $\begin{array}{c}\text { Matériels } \\
\text { aux laboratoires } \\
\text { extérieurs }\end{array}$ & $\begin{array}{c}\text { Matériels } \\
\text { aux laboratoires } \\
\text { CNRS }\end{array}$ & $\begin{array}{c}\text { Achats } \\
\text { à l'étranger }\end{array}$ & Missions & $\begin{array}{c}\text { Ensemble } \\
\text { Publications (1) }\end{array}$ \\
\hline 1946 & 71 & 39 & 20 & 9 & \\
\hline 1947 & 88 & 90 & 18 & 14 & 28 \\
1948 & 83 & 93 & 12 & 18 & 36 \\
\hline 1949 & 137 & 117 & 31 & 38 & 70 \\
\hline 1950 & 148 & 68 & 31 & 33 & 82 \\
1951 & 180 & 141 & 26 & 46 & 112 \\
1952 & 250 & 210 & 30 & 57 & 149 \\
1953 & 260 & 210 & 33 & 65 & 166 \\
1954 & - & 257 & - & - & 162
\end{tabular}

(1) inclut publications du CNRS, contrats d'édition et subventions pour publications.

7 Au regard des chiffres disponibles, les dépenses ordinaires augmentent constamment au cours de la période, même s'il s'agit d'une croissance par à-coups. Les crédits d'équipements connaissent au contraire une diminution drastique dès 1945. Ce qui peut apparaître comme un accident lié à des circonstances extraordinaires se répétera pourtant à maintes reprises dans l'histoire financière de l'institution. Les données sont trop lacunaires entre 1949 et 1956 pour savoir si le programme d'extension des laboratoires décidé le 30 septembre 1948 par le CNRS a été suivi d'un soutien budgétaire réel et régulier. Cependant, les chiffres disponibles à partir de 1956 indiquent un véritable bond par rapport à 1951 des crédits d'équipement, date de la dernière subvention connue avec certitude. Trois domaines scientifiques sont d'emblée privilégiés par les investissements et sont appelés à devenir les « masses de granit » de l'activité scientifique au CNRS : ce sont l'astrophysique, les sciences de la vie et la physique. Malgré ce programme d'investissement sans précédent, les dépenses extraordinaires représentent à peu près la moitié des dépenses ordinaires aux deux extrémités de la période. Cette disproportion entre les deux budgets constitue également une autre tendance lourde du Centre, qui ne sera pas sans effet sur le soutien consacré à son activité scientifique.

\section{La manne financière des années soixante (1959-1968)}

8 La réforme de 1959 n'a pas seulement pour objet d'organiser la recherche publique et de redéfinir les missions du Centre. Pour être pleinement efficace, elle est accompagnée 
d'un soutien financier exceptionnel, rendu possible par les années de haute croissance et par un pouvoir politique qui a fait de la recherche fondamentale l'une des grandes priorités du nouveau régime. L'ambition du pouvoir gaullien est bien de ramener l'effort de recherche français au niveau de celui des grandes puissances occidentales par rapport auxquelles il a pris du retard. Alors qu'en 1962, les Etats-Unis consacrent à la R-D 3, 1 \% de leur PNB et le Royaume-Uni 2, $2 \%$, la France n'y investit que 1, $5 \%$ du sien $^{6}$.

9 Exprimé en nouveaux francs, le budget du Centre passe de 173 millions de francs courants en 1959 à 754 millions en 1968. Cette augmentation correspond à un bond de $335 \%$ du budget en l'espace de neuf années, soit une hausse moyenne annuelle de $17,6 \%$ en francs courants. Si l'on annule l'effet inflation, l'accroissement moyen annuel est encore de l'ordre de 13,6\%. Cependant, ce dernier chiffre demeure quelque peu en deçà de la croissance moyenne annuelle de la période antérieure (1944-1958) qui était de l'ordre de 15,7\%. En fait, l'originalité de la phase 1959-1968 réside surtout dans la plus grande régularité d'élévation des crédits accordés au Centre. La grande différence entre les deux périodes est donc autant quantitative que qualitative: le CNRS des années soixante bénéficie d'un budget en hausse constante, toujours forte mais plus régulière. Ces caractéristiques budgétaires sont certes la conséquence heureuse de la croissance économique et des choix politiques du pouvoir gaulliste. Elles sont aussi le résultat d'un nouveau dispositif susceptible d'améliorer la distribution et la répartition des deniers publics, à savoir l'enveloppe recherche, dont l'inspirateur fut Pierre Piganiol.

\section{Budget du CNRS de 1944 à 1969}

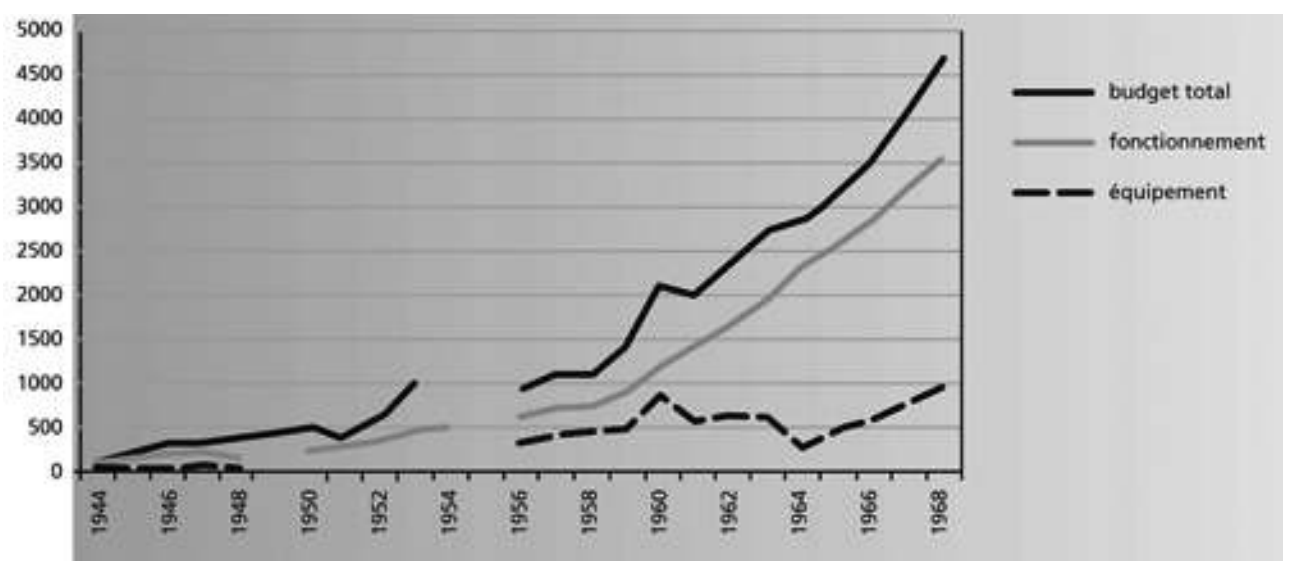

(en millions de francs 1999)

10 Au regard de l'effort global de recherche français, public et privé, exprimé en dépense brute de recherche-développement (DBRD), le CNRS représente une proportion de l'ordre de $5 \%$ entre 1963 et 1968 . Cependant, sa position tend à s'affaiblir au milieu de la décennie dans la mesure où sa part passe de $5,7 \%$ en 1963 à $4,7 \%$ en 1966, pour remonter à 5, $5 \%$ en 1968. Si l'on compare l'évolution des subventions publiques reçues par le CNRS avec le budget de l'Etat consacré à la recherche-développement, il apparaît que la position du CNRS se renforce sensiblement de 1959 à 1960, de 8, 7 \% à 11, $6 \%$, mais s'amoindrit dans les années suivantes, passant à 7, $8 \%$ en 1963. Une évolution similaire se dessine si l'on rapporte la part des subventions du Centre à l'ensemble de l'enveloppe recherche. Il reçoit $61,4 \%$ puis $62,5 \%$ des crédits en 1959 et 1960, mais là 
aussi sa part décroît inexorablement à partir de 1961 pour se réduire à moins de la moitié de l'enveloppe à partir de 1963. Cependant, après un étiage de 38, 5 \% atteint en 1964, la part du CNRS remonte à $47 \%$ en 1966. Au final, si le montant de l'enveloppe recherche fait plus que quadrupler entre 1959 et 1966, celui des subventions que reçoit CNRS est multiplié par un peu plus de trois. De ces diverses comparaisons, il ressort que le CNRS est loin de bénéficier d'une position privilégiée au sein du dispositif de financement public de la recherche-développement.

11 L'évolution comparée des subventions étatiques témoigne certes d'un affaiblissement relatif du Centre par rapport aux autres structures financées comme lui au moyen de l'enveloppe recherche, mais ses laboratoires sont désormais mieux dotés en moyens financiers et humains. Comme le souligne le rapport Verdeil de 1962, les laboratoires propres du CNRS absorbent alors $62 \%$ des crédits de fonctionnement et les deux tiers des crédits d'équipement. Un chercheur y dispose en moyenne de quatre techniciens et de 100000 francs de crédits de fonctionnement, contre un demi technicien et moins de 20000 francs pour son collègue d'une faculté de science ${ }^{7}$. C'est un renversement de priorité notoire par rapport à la période précédente. Le «CNRS-institut » tend à supplanter le «CNRS-caisse ».

12 La structure budgétaire du CNRS tend, en revanche, à respecter les équilibres antérieurs. Les années de reconstruction avaient signalé la prédominance du budget de fonctionnement sur des crédits d'équipement dont l'évolution s'était avérée plus erratique. Ces tendances se confirment durant la décennie 1960. Ainsi la part du premier budget quadruple presque tandis que celle du deuxième double à peine. De plus, les crédits d'équipement affichent toujours de fortes distorsions d'une année sur l'autre, ce qui n'est pas le cas des crédits de fonctionnement. Cette différence de traitement est liée pour partie aux particularités du système budgétaire français : la soumission à révision complète est limitée aux seuls crédits d'équipement, tandis que pour le budget de fonctionnement seules sont discutées les augmentations demandées. $\mathrm{Au}$ final, le budget de fonctionnement peut, dans le pire des cas, ne pas augmenter, mais les subventions pour équipement risquent d'être l'objet d'une profonde amputation. Néanmoins, de 1965 à 1968, les crédits d'équipement sont plus enclins à prendre la relève de la dynamique budgétaire. La plus faible croissance des crédits de fonctionnement peut s'expliquer par la moindre pression des dépenses de personnel dont la part diminue tout au long de la décennie. Après avoir atteint un pic en 1959, cette proportion décroît d'une dizaine de points pour être ramenée à $71,5 \%$ du budget de fonctionnement en fin de période. Le recrutement du CNRS s'établit à un rythme de l'ordre de $+15,4 \%$ par an en moyenne, pour atteindre un effectif de 14761 personnes en 1968, rythme deux fois moins élevé cependant qu'au cours de la période antérieure.

Une innovation juridique permet également au fonctionnement du Centre de gagner en souplesse. L'une des dispositions majeures de la réforme du 31 mai 1966 concerne en effet l'introduction d'une « ligne souple » dont l'usage est laissé à la discrétion de la direction de l'établissement, qui dispose de fait d'une latitude d'action supérieure ${ }^{8}$. La « ligne souple » devient ainsi un levier essentiel des nouveaux attributs de la direction qui est sortie renforcée des décrets de 1966. Le Centre relève toujours du droit commun mais des dérogations sont possibles. Sur ce point, la situation du CNRS se révèle plus favorable que celle de la plupart des autres établissements administratifs, qu'ils soient des organismes de recherche ou non. Cette "ligne souple» est destinée, comme le mentionne le rapport d'activité de 1966, à couvrir des actions spécifiques de recherche 
ou bien à renforcer accessoirement en cours d'année tel ou tel chapitre de dépenses. L'assouplissement des règles financières du CNRS ouvre la voie à la création des instituts nationaux qui disposeront de l'autonomie budgétaire. L'INAG est créé l'année suivante, par décret du 11 septembre 1967. Il dispose, lors de sa première année de fonctionnement, d'un modeste budget de 5,3 millions de francs, qui s'élève néanmoins à 28 millions dès 1969. La souplesse budgétaire amorcée dans le mitan des années 1960 annonce aussi la vague des nouvelles expériences de gestion du Centre étrennées à l'extrême fin de la décennie.

\section{NOTES}

1. Sources utilisées pour reconstituer le budget dans cet article : rapports d'activité, procèsverbaux de conseils d'administration, série de cartons consacrés au budget et conservés au dépôt de Gif-sur-Yvette (G 990001 ART 15 à 21). Des sources secondaires sont intervenues en appui, soit pour combler les lacunes des sources primaires, soit pour obtenir des informations complémentaires, notamment les diverses séries statistiques dressées par les services centraux à partir des années 1970,

2. Le budget du CNRS est présenté en francs constants de 1999, qui correspond à la date terminale de notre étude. Nous avons utilisé la table de conversion établie par Michel-Pierre Chélini, présentée en annexe de son Histoire du franc au XX $X^{e}$ siècle, Paris, Picard, 2001, p. 363-364.

3. Séance plénière du 2 juin 1948.

4. Les dénominations varient constamment pour désigner les deux grandes masses budgétaires du CNRS. Au cours des années 1970 s'impose la distinction entre "fonctionnement " et " équipement». L'instruction du 25 février 1968 précise que «les crédits d'équipement (ou dépenses en capital) sont destinés à l'acquisition de tout bien dont la fonction et la durée d'utilisation lui permettent de rester durablement et sous la même forme à la disposition du CNRS.

5. P.V. de la séance du conseil d'administration du 16 février 1950. Le SMIG a été instauré par la loi du 11 février.

6. La France fait néanmoins légèrement mieux que la RFA (1, 3 \%). Cf. C. Freeman et A. Young, L'Effort de recherche et de développement en Europe occidentale, Amérique du Nord et Union soviétique Essai de comparaison internationale des dépenses et des effectifs consacrés à la recherche en 1962, OCDE, 1965, p. 11-12.

7. G. Verdeil, « Note sur certains problèmes posés par la répartition des crédits du Centre national de la recherche scientifique », 16 juillet 1962. A.N. 77 / 321-323, liasse 894.

8. Cette décision fut entérinée à la suite du Conseil interministériel du 19 mars 1965 et du Conseil restreint du 25 mai 1965. 


\section{RÉSUMÉS}

«Mais qu'allons nous faire de tout cet argent?». Quel organisme public pourrait se prévaloir de tels mots en ces temps actuels de rigueur? Cette phrase fut pourtant bien prononcée, en 1959, par un administrateur du CNRS, à l'annonce du prochain budget. Bruno Marnot analyse ici l'évolution du budget du CNRS - globalement en hausse sur toute la période étudiée - qu'il replace dans le contexte d'une économie florissante, couplée à une politique volontariste en faveur de la Recherche avec l'arrivée du Général de Gaulle à la tête du pays. Il explique également l'évolution de l'utilisation par le CNRS des budgets qui lui sont alloués, tout en repositionnant le CNRS dans le cadre plus général de la Recherche française d'alors.

"What shall we do with all this money?". Which government agency would rely on such words in these current times of economic stringency? This sentence was however pronounced in 1959 by a director of the CNRS as the next budget was announced. Bruno Marnot analyses the budgetary developments of the CNRS - which increased all over the studied period. He puts this development back in the context of a booming economy, coupled with a proactive policy for Research by General de Gaulle once he had been elected President. Bruno Marnot also explains the evolution of the use by the CNRS of its allocated budgets, while repositioning the CNRS in the broader context of French Research at that time.

\section{AUTEUR}

\section{BRUNO MARNOT}

Docteur en Histoire de l'Université Paris IV Sorbonne, Bruno Marnot est actuellement maître de conférences à l'Université Michel de Montaigne Bordeaux III et chargé de cours à l'IEP de Bordeaux. 